\title{
Spatiotemporal Analysis of Seawatch Buoy Meteorological Observations
}

\author{
A. K. Charakopoulos ${ }^{1}$ - T. E. Karakasidis ${ }^{1}$ • \\ A. Liakopoulos ${ }^{1}$
}

Received: 28 January 2015 / Accepted: 17 July 2015 /Published online: 14 August 2015

(C) Springer International Publishing Switzerland 2015

\begin{abstract}
In the present study, we analyzed meteorological observations from Seawatch buoys in the Mediterranean Sea and specifically from locations in the Aegean and Ionian Sea. The data were collected from buoys that have been deployed by the Hellenic Center for Marine Research (HCMR) in the framework of the POSEIDON project. Our aim was to understand the spatiotemporal underlying characteristics of the meteorological conditions and identify correlation patterns between different locations. For each time series at a given buoy location we estimated mainly non-linear measures such as mutual information combined with simple descriptive statistic measures, as well as some other dynamics detectors such as Hurst Exponent and Hjorth parameters. Furthermore, the relationships between the meteorological variables were investigated using the cross correlation method as well as Granger causality methodology for identification of direction interactions among variables. The results show that the combination of the proposed methods reveals information about the spatiotemporal characteristics of the time series.
\end{abstract}

Keywords Spatiotemporal analysis · Seawatch buoys · Cross correlation · Granger causality Hurst exponent

\section{Introduction}

Analysis of spatiotemporal observations is of major importance for scientists due to spatial and also temporal variability of the observable physical quantities. Meteorological observations due to their spatial dependence and temporal variation are characterized as spatiotemporal data. Analysis of data, such as air temperature, precipitation, chlorophyll, salinity etc. is crucial for understanding the underlying processes and improving the prediction of climate changes (Gaur and Simonovic 2015; Meiling et al. 2014; Paruch et al. 2014; Darand and Mansouri Daneshvar

\section{A. K. Charakopoulos avracha@yahoo.gr}

1 Laboratory of Hydromechanics and Environmental Engineering, Department of Civil Engineering, University of Thessaly, 38334 Volos, Greece 
2014; Bagiorgas et al. 2012; Craigmile and Guttorp 2011; Pasini et al. 2012; Sigrist et al. 2012; Drakopoulos et al. 2011; Tastu et al. 2011; Triacca 2005; Nittis et al. 2001a, b; Wilks 1995).

Over the last decades, due to the fact that the distribution and changes of the sea and oceanic variables is a major component in the hydrological cycle, the analysis by scientists using data from buoys focused on the temporal and spatial variability of general circulation structures, and on understanding the basic characteristic mechanism that occur in the examined basin. More specifically, research has been focused on the general circulation of the basin, the water mass formation processes, and the effects of these mechanisms on the biochemical cycles at seasonal time scales in order to design a reliable efficient system that could be useful for predicting atmospheric and oceanographic variables.

Several buoy systems have been developed such as the Poseidon system which is a monitoring, forecasting and information system for the Hellenic Seas and Coastal areas, operated by the Hellenic Center for Marine Research (HCMR) providing real time information and forecasts for a large number of observations (Soukissian et al. 1999). Several numerical investigations have been realized using data series provided by the Poseidon system (Kassis et al. 2013; Velaoras et al. 2013; Bagiorgas et al. 2012; Kopasakis et al. 2012; Korres et al. 2011; Papadopoulos and Katsafados 2009; Papadopoulos et al. 2002; Nittis et al. 2001a, b).

The data used in this work were provided from the Poseidon system mentioned above. The data were collected from sea watch buoys which are equipped with meteorological and oceanographic sensors. In this work, we utilized time series measured from Athos (Aegean Sea) and Pylos (Ionian Sea) stations which have differences in atmospheric and hydrodynamic characteristics. These locations are part of the Eastern Mediterranean Sea where water masses of different basin interact and transform at various temporal and spatial scales.

Since the knowledge of meteorological parameter interactions, can help to better understand the cause-effect mechanisms in climate science, the objective of the present work was to investigate such mechanisms with an alternative view focused on the relationships among physical variables. Additionally, this work examines relationships and patterns at the microscale level (locally at each station) as well as at macro-scale level (globally between the stations) using a range of methods from linear to nonlinear measures (Kugiumtzis 2013; Papana et al. 2013; Paluš 2014; Probst et al. 2012; Nelson-Wong et al. 2009). First, the cross correlation analysis among variables was performed to study the correlations between the pairs of variables as a function of the time lag. Since the existence of correlation between two variables does not necessarily demonstrate the direction of causality, we applied Causality analysis in order to test the significance of causal relationships between specific variables. In this way, we applied the Granger Causality method, which has already been successfully applied to a wide range of data from various fields of social science, economics, engineering and neurosciences, to identify the presence of linear causal interactions between variables, based on prediction theory (Papana et al. 2013; Attanasio et al. 2013; Pasini et al. 2012; Attanasio et al. 2012; Bilancia and Vitale 2012; Kodra et al. 2011; Seth 2010).

Also, our analysis includes, for each variable from Athos and Pylos station, the performance of non-linear measures such as mutual information combined with simple descriptive statistic measures. Furthermore, we estimated the Hurst Exponent and the Hjorth parameters which are additional dynamic detectors (Hjorth 1970). The results show that there are correlations and causalities between variables, both from the same buoy and also between different spatial buoys and the performance of these methods may uncover hidden qualitative differences between variables which cannot be determined from the simple statistical analysis. 
The remainder of this paper is organized as follows. In Section 2, we portray the site and data description. In Section 3, we present the methodology employed. The results and discussion are presented in Section 4. Finally, the conclusions are presented in Section 5.

\section{Site and Data Description}

The data are collected from buoys that have been developed by the Hellenic Center for Marine Research (HCMR) in the framework of the Poseidon project (www.poseidon. hcmr.gr) (Soukissian et al. 1999). The Poseidon system is an operational monitoring, forecasting and information system for the Hellenic seas, providing in real time information and forecasts for a large number of observations. The system consists of a network of 10 oceanographic buoys installed in the Aegean and Ionian Sea. The buoys are equipped with meteorological, oceanographic sensors which collect meteorological data, such as atmospheric pressure, air temperature, wind speed and direction, physical data such as salinity, water temperature, and biochemical data such as chlorophyll and dissolved oxygen.

In this work, we employed data from monitoring buoys located at Athos (Aegean Sea) and Pylos (Ionian Sea), and cover a period from January 2011 to November 2012 which corresponds to a duration of 23 months. The choice of the given sites was based on the fact that these basins present different physical characteristics. The major feature of the Aegean Sea is the existence of many straits connecting the basin with the rest Eastern Mediterranean and Black Sea as well as the Cyclades islands that separate the Aegean in two basins. The intrusion through the Dardanelles Straits of water mass interacts with the main water. The Ionian Sea is characterized by complex hydrology where different water masses of Western Mediterranean and Adriatic Sea interact and also connect to the Aegean Sea through the strait lying between the Peloponnese peninsulas (Velaoras and Lascaratos 2010; Kassis et al. 2013).

In Table 1, we summarize the latitude, longitude, the data collection period and the collected observations for each station, and the physical locations are depicted in Fig. 1.

Atmospheric Pressure (Atm. P.), Air Temperature (Air T) and Wind Speed (Win. S) were recorded at $3 \mathrm{~m}$ height above sea level while Water Temperature (Wat. T) at $3 \mathrm{~m}$ depth. The sampling interval was $3 \mathrm{~h}$ for all variables and every measurement is an average over $10 \mathrm{~min}$, resulting in 8 values per day. Thus, the length of the time series is 5.600 values (in units of $\Delta t=$ 3 h). We used the Piecewise Cubic Hermite Interpolating Polynomial as an interpolation

Table 1 Main Parameters and measurement variable of the stations

\begin{tabular}{|c|c|c|c|c|c|}
\hline \multirow{2}{*}{$\frac{\text { Location }}{\text { Athos }}$} & \multirow{2}{*}{$\begin{array}{l}\text { Latitude } \\
39^{\circ} 57^{\prime} .843 \mathrm{~N}\end{array}$} & \multirow{2}{*}{$\begin{array}{l}\text { Longitude } \\
24^{\circ} 43^{\prime} 208^{\prime} \mathrm{E}\end{array}$} & \multicolumn{2}{|c|}{ Measurement period } & \multirow{2}{*}{$\begin{array}{l}\text { Observations } \\
\text { Atmospheric pressure } \\
\text { Air temperature } \\
\text { Water temperature } \\
\text { Wind speed }\end{array}$} \\
\hline & & & $01 / 2011$ & $11 / 2012$ & \\
\hline Pylos & $36^{\circ} 49^{\prime} .533 \mathrm{~N}$ & $21^{\circ} 35^{\prime} 762 \mathrm{E}$ & $01 / 2011$ & $11 / 2012$ & $\begin{array}{l}\text { Atmospheric pressure } \\
\text { Air temperature } \\
\text { Water temperature } \\
\text { Wind speed }\end{array}$ \\
\hline
\end{tabular}




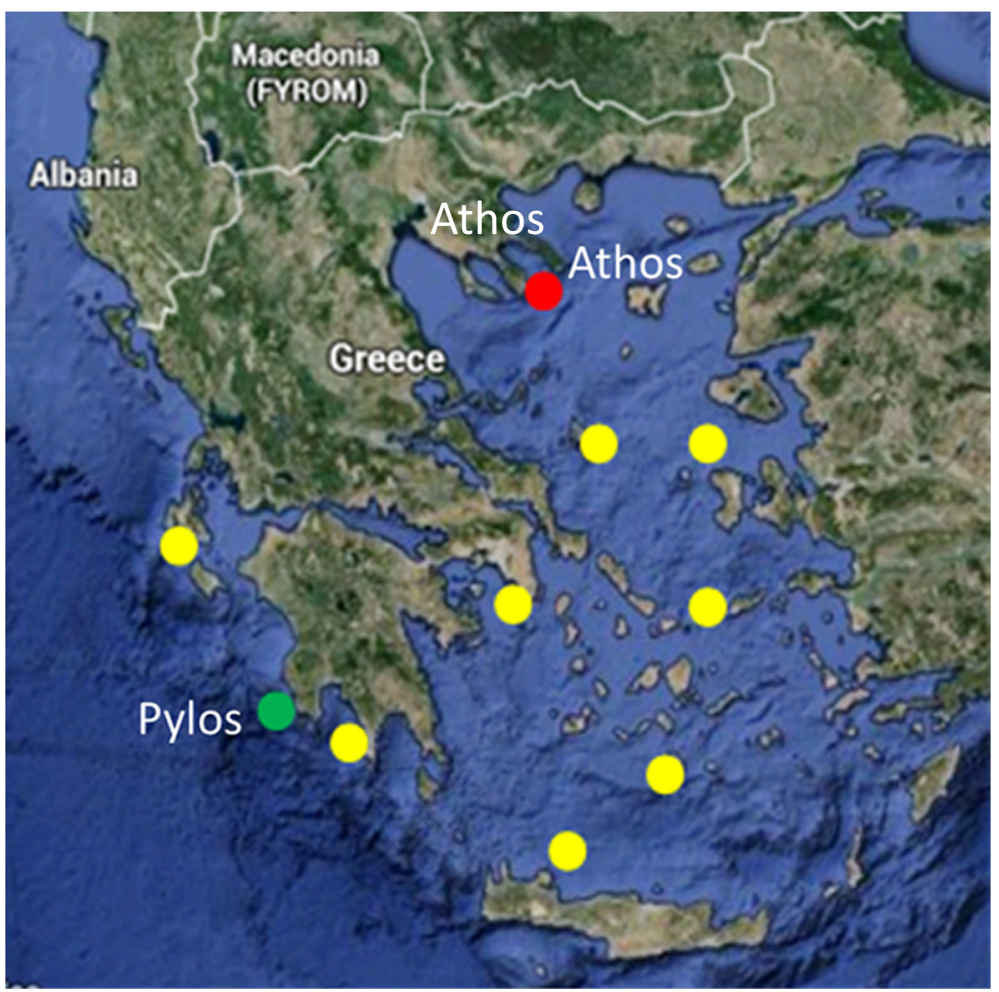

Fig. 1 Locations of Poseidon network buoys. The red cycle indicates Athos station while the green cycle corresponds to Pylos station. Yellow cycles present the location of the remaining boys in the Aegean and Ionian Seas

method (Fritsch and Carlson 1980) to fill the missing values which occurred either as a result of transmission or power problems or due to maintenance of the sensors. Also, extreme values, spikes and obviously wrong values were removed.

\section{Methodology}

One of the major challenges of the researchers is to identify and analyze patterns in global climate in order to understand the interactions between physical variables. Climate scientists often want to understand mechanisms on a micro (local) level and a macro (global) level scale. The methodology to study the spatiotemporal variation of meteorological observations includes estimation of non-linear measures such as mutual information combined with conventional descriptive statistic measures, as well as linear and nonlinear dynamic detectors such as Hurst Exponent and Hjorth parameters. The cross correlation analysis between all possible pairs of variables both at local scale (each station) and global scale (between the two stations) was performed. Furthermore, the cause-effect relationships between the meteorological variables was examined using the Granger causality methodology. 


\subsection{Descriptive Statistics}

We performed conventional descriptive analysis and in order to summarize and describe our data set. More specifically, we estimated the mean, median, variance, standard deviation and kurtosis of each time series.

\subsection{Mutual Information}

For two random variables Mutual Information $I(t)$ is a quantitative measurement of the amount of information that one variable contains about another (Fraser and Swinney 1986). In the time series analysis it is used to measure any form of linear and nonlinear relations and is the most popular measure used to select the appropriate delay time $\tau$ for state space reconstruction. Mutual information between elements of a time series $x\left(t_{i}\right)$ is defined as:

$$
I(\tau)=\sum_{x\left(t_{i}\right), x\left(t_{i}+\tau\right)} P\left(x\left(t_{i}\right), x\left(t_{i}+\tau\right)\right) * \log \left[\frac{P\left(x\left(t_{i}\right), x\left(t_{i}+\tau\right)\right)}{P\left(x\left(t_{i}\right) * P x\left(t_{i}+\tau\right)\right)}\right],
$$

where $x\left(t_{i}\right)$ is the $\mathrm{i}$-th data point of time series, $P\left(x\left(t_{i}\right)\right)$ is the probability density at $x\left(t_{i}\right)$, $P\left(x\left(t_{i}\right) x\left(t_{i}+\tau\right)\right)$ is the joint probability density at $x\left(t_{i}\right), x\left(t_{i}+\tau\right)$ and $\tau$ is the delay time.

\subsection{Estimation of Hurst Exponent}

In the time series analysis, Hurst exponent $(H)$ is a measure of long range correlation which exhibits properties of self similarity. Estimation of the Hurst exponent was firstly reported in hydrology by Hurst (1951) while investigating the discharge time series of the Nile River and subsequently has been used in diverse areas of research such as climate science, geophysics and economics just to mention a few. The oldest method to calculate the Hurst exponent, called Rescaled Range or $R / S$ analysis, was proposed by Mandelbrot and Wallis (1969) and estimates of $H$ are based on the R/S statistics.

Suppose we have a time series of $\mathrm{N}$ measurements. We divide the time series into $N_{s}$ shorter subseries of length $n=N, N / 2, N / 4$. For each subseries we define the range $R_{n}$

$$
R_{n}=\max _{1 \leq k \leq s}\left[\sum_{i}^{k}\left(x_{n s+i}-\overline{x_{n}}\right]-\min _{1 \leq k \leq s}\left[\sum_{i}^{k}\left(x_{n s+i}-\overline{x_{n}}\right],\right.\right.
$$

where $=0,1, \ldots \ldots N_{s}-1, N_{s}=N / S$ and

$$
\overline{x_{n}}=\frac{1}{s} \sum_{i=1}^{s} x_{n s+i}
$$

The sample standard deviation is defined as

$$
S_{n}=\sqrt{\frac{1}{s} \sum_{i=1}^{s}\left(x_{n s+i}-\overline{x_{n}}\right)^{2}} .
$$


Then, the rescaled range is defined as the ratio $R_{n} / S_{n}$. The Hurst exponent is estimated by calculating the average rescaled range for all subseries of length $n$. It can be shown that the $R / S$ statistics follows the relation

$$
(R / S)_{n}=c \cdot n^{H} \quad \text { as } \quad n \rightarrow \infty .
$$

Hence, we can plot the diagram $\log (R / S)_{n}$ vs $\log (\mathrm{n})$ and we calculate the Hurst exponent using linear least squares regression. With this definition, the Hurst exponent of 0.5 indicates a random series, when a value of $H$ lying within the interval $0.5<H<1$ indicates persistent behaviour (i.e., a large value is followed by a large value, a small value by a small one), while a Hurst exponent value between 0 and 0.5 exists for time series with anti-persistent behaviour (i.e., a large value is followed by a small value, a small value is followed by a large value).

\subsection{Hjorth Parameters}

The Hjorth descriptors, namely activity, mobility and complexity, were mainly developed for quantification of an Electroencephalogram (EEG) by Hjorth (1970). The Hjorth parameters for a given time series $x(t)$ are called normalized slope descriptors because they can be defined as first and second derivatives and are respectively defined as follows:

$$
\begin{gathered}
\text { Activity }=m_{o} \\
\text { Mobility }=\sqrt{\frac{m_{2}}{m_{0}}} \\
\text { Complexity }=\sqrt{\frac{m_{4} / m_{2}}{m_{2} / m_{0}}},
\end{gathered}
$$

where $m_{0}$ is the variance (square of the standard deviation) of the variable, $m_{2}$ is the variance of the first derivative of the variable, and $m_{4}$ is the variance of the second derivative of the variable.

Activity is a measure of the squared standard deviation of the amplitude and represents the width of the signal. Mobility is defined as the squared root of the ratio between the variances of the first derivative and the amplitude, and is related to the mean frequency of the time series. Complexity is the ratio between the mobility of the first derivative and the mobility of the nonlinear time series. Complexity indicates the deviation of the slope and can be seen as a measure of change in frequency of the signal.

\subsection{Cross Correlation}

Cross correlation analysis ( Nelson-Wong et al. 2009; Probst et al. 2012) is a statistical tool that measures the simultaneous linear coupling relationship between two time series $x(t)$ and $y(t)$ that are out of the phase with a lag time $t$. It calculates the correlation between time series, when shifted against one another as a function of number of observations. The shape of the cross correlation coefficient curve versus time represents the variation of the correlation among 
the two time series depending on the lag time. However, we must mention that the presence of correlation does not imply necessarily causality.

The cross correlation between $X_{t}$ and $Y_{t^{+} k}$ is called the $k_{t h}$ order cross correlation of $X$ and $Y$. The sample estimate of this cross correlation, called $r_{k}$, is calculated using the formula:

$$
\begin{gathered}
r_{k}=\frac{\sum_{i=1}^{n-k}\left(X_{i}-\bar{X}\right)\left(Y_{i+k}-\bar{Y}\right)}{\sqrt{\sum_{i=1}^{n}\left(X_{i}-\bar{X}\right)^{2} \sum_{i=1}^{n}\left(Y_{i}-\bar{Y}\right)^{2}}} \\
\bar{X}=\frac{1}{n} \sum_{i=1}^{n} X_{i} \\
\bar{Y}=\frac{1}{n} \sum_{i=1}^{n} Y_{i}
\end{gathered}
$$

The time index $k$, is allowed to be either positive or negative. The large sample standard error of the sample cross correlations is simply $1 / \sqrt{N}$ and $2 / \sqrt{N}$ so that large sample confidence limits are $\pm 2 / \sqrt{N}$ (at the $5 \%$ level). If the above is computed for all delays $d=$ $0,1,2, \ldots N-1$ then it results in a cross correlation series of twice the length as the original series.

\subsection{Granger Causality}

Identifying causal connectivity between meteorological variables is an important issue. Granger Causality analysis is one of the most popular computational approaches, that has already been applied with great success in a range of fields such as social sciences, financial, computational, engineering, neuroscience and environmental sciences in order to assess causality among variables. First, Wiener (1956) introduced the idea that if the prediction of one time series can be improved by incorporating the knowledge of a second time series, then the latter can be said to have a causal influence on the first. Granger (1969) implemented this idea in the context of linear autoregressive models of stochastic processes.

Let us suppose that we have two stochastic processes $X_{t}$ and $Y_{t}$ with autoregressive representations in the form:

$$
\begin{aligned}
& X_{t}=\sum_{j=1}^{\infty} a_{1 j} X_{t-j}+\sum_{j=1}^{\infty} b_{1 j} Y_{t-j}+\varepsilon_{t} \\
& Y_{t}=\sum_{j=1}^{\infty} c_{1 j} X_{t-j}+\sum_{j=1}^{\infty} d_{1 j} Y_{t-j}+\eta_{t}
\end{aligned}
$$


where $a, b, c$ and $d$ are coefficients describing the linear interactions between the variables, $\varepsilon$ and $\eta$ are residuals (prediction errors) for each time series and $j$ indicating the time lags.

The covariance matrix of the noise terms is:

$$
\Sigma=\operatorname{Cov}\left(\begin{array}{c}
\varepsilon_{t} \\
\eta_{t}
\end{array}\right)
$$

If the variance of $\varepsilon$ (or $\eta$ ) is reduced by the inclusion of the $X_{t}$ (or $Y_{t}$ ) terms in the first (or second) equation, then it is said that $X_{t}$ (or $Y_{t}$ ) Granger-(G)-causes $X_{t}$ (or $Y_{t}$ ). In other words, $X_{t}$ G-causes $Y_{t}$ if the coefficients in $b_{1 j}$ are jointly significantly different from zero. We used the Conditional Granger Causality methods described in the 'Multivariate Granger Causality Toolbox' (MVGC) by Barnett and Seth (2014).

\section{Results and Discussion}

The possibilities of extracting useful information about the meteorological characteristics of the Aegean and Ionian Sea through the analysis of observation from Seawatch buoys are examined. In Fig. 2a to d, we plot the time series of Atmospheric Pressure (Atm. P), Air Temperature (Air. T), Wind Speed (Win. S) and Water Temperature (Wat. T) of Athos and Pylos as recorded. The horizontal axis refers to time $(t)$ and the vertical axis refers to each measurement variable. Seasonal variability is prominent in Fig. $2 \mathrm{~b}$ and $\mathrm{d}$.
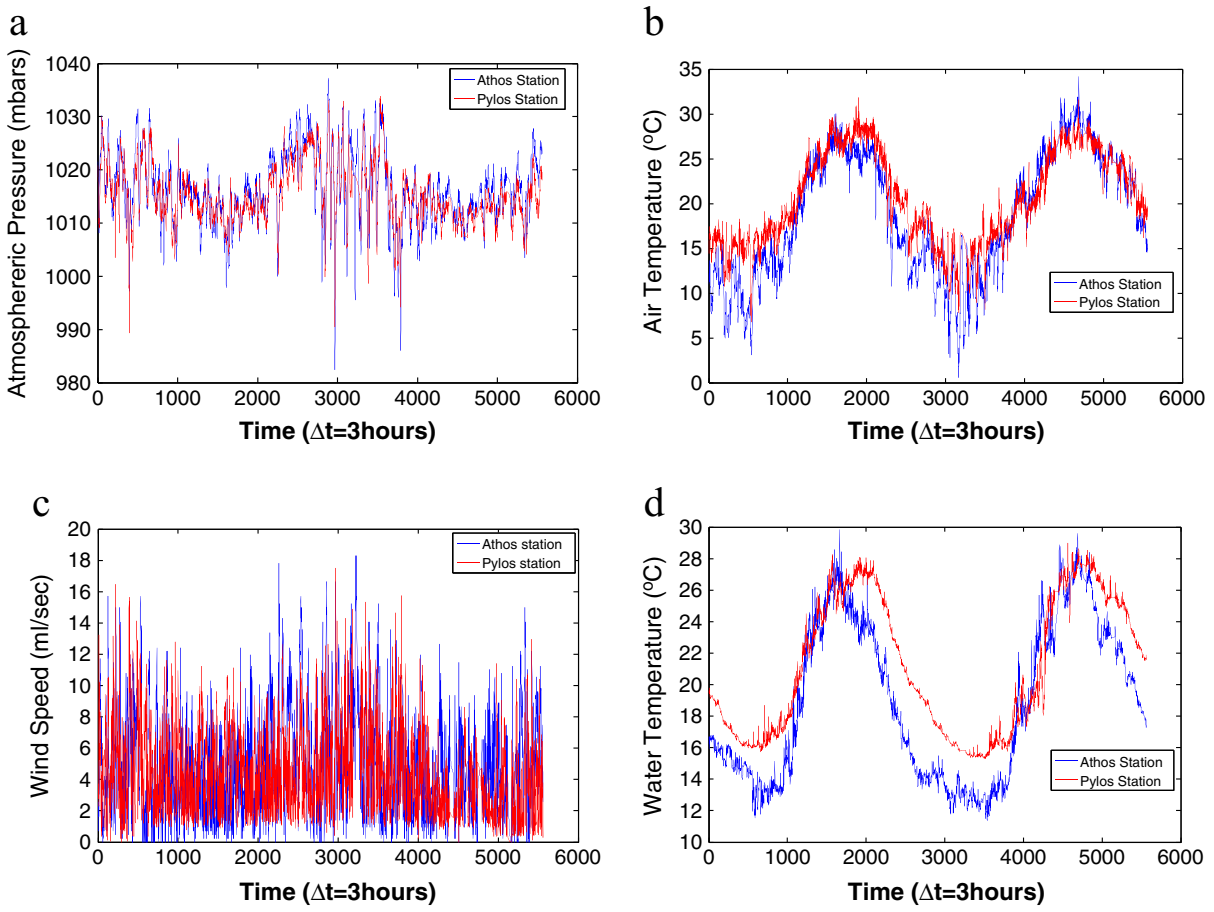

Fig. 2 Time series of Atmospheric Pressure (a), Air Temperature (b), Wind Speed (c) and Water Temperature (d) of the Athos Station (blue line) and Pylos Station (red line) (time is given in units $\Delta \mathrm{t}=3 \mathrm{~h}$ ) 
As we have mentioned before for each time series, we first calculated the simple statistical measures, i.e., the mean, median, variance, standard deviation, and kurtosis. The objective of this part is to study the performance of simple statistical estimators and check whether it is possible to identify patterns among variables. The results of mean, median variance, standard deviation and kurtosis are summarized in Table 2. As we can clearly observe, the values of variance, standard deviation and kurtosis of Pylos station for all measured variables are lower than those of Athos station, indicating the differences in the hydrodynamic characteristics of the two locations. Specifically, from the results of mean and median of Atmospheric pressure variable, both from Athos and Pylos stations the values are nearly identical, indicating that the Atm. P. variable has no significant difference between the stations. Also, at a global level for Air Temperature and Water Temperature variables, comparing the results of mean and median, we can see that they are higher at Pylos station than at Athos station. This is evidence which indicates the different meteorological and hydrodynamics conditions of the two basins (Aegean and Ionian) (Velaoras and Lascaratos 2010; Kassis et al. 2013).

Then, we evaluated the mutual information, with the aid of Eq. (1), which detects linear and nonlinear dependencies between time series. In order to compare the estimated mutual information from different variables, in Fig. 3 we present the normalized values of $I(\tau)$.

A first remark from Fig. 3 is that the behavior of the mutual information function is nearly identical for each variable at both the Athos and Pylos station. Also, from Fig. 3 it can be seen that for any value of time delay the mutual information function of the Win. S. of Athos and also Pylos station clearly attains the lowest values. This behavior is expected since wind speed varies more frequently, as seen in Fig. $2 a-d$ than all the other quantities and affects more directly pressure than temperature. Pressure presents a slower decay than wind speed, which means that the values of the time series of the Atm. P. are less independent in all time lags than the values of the time series of Win. S. This is constant with the physical interpretation as we can also see from the Fig. $2 \mathrm{a}$ and $\mathrm{d}$ the Atm. P values varies less frequently than the values of Win. S. The Air. T time series present higher correlations than Win. S and finally the higher values (for any value of time delay) are observed for the Wat. T time series. Mutual information values of Air T. time series and Wat. T time series seem to have also a kind of periodic behavior with a maximum around 1 day ( 8 values per day). We have to mention that Air temperature presents lower mutual information values than water temperature since air temperature changes faster than temperature water (we remind that water has a significantly higher specific heat than air).

Next, we estimated the linear and nonlinear dynamic detectors, i.e., the Hurst exponent and Hjorth parameters. The evaluation of Hurst exponent for each time series with $R / S$ method is shown in Table 3. The Hurst exponents of the observations from both Athos and Pylos stations

Table 2 Descriptive statistics measures

\begin{tabular}{lllllllll}
\hline & $\begin{array}{l}\text { Athos } \\
\text { Atm. P }\end{array}$ & Pylos & $\begin{array}{l}\text { Athos } \\
\text { Air. T }\end{array}$ & Pylos & $\begin{array}{l}\text { Athos } \\
\text { Wat. T }\end{array}$ & Pylos & $\begin{array}{l}\text { Athos } \\
\text { Win. S }\end{array}$ & Pylos \\
\hline Mean & 1016.26 & 1015.42 & 18.62 & 20.66 & 18.85 & 21.14 & 5.01 & 4.52 \\
Median & 1015.70 & 1014.70 & 18.24 & 20.05 & 18.28 & 20.14 & 4.41 & 4.10 \\
Variance & 43.92 & 31.31 & 46.54 & 26.29 & 24.30 & 18.17 & 10.73 & 7.73 \\
Std deviation & 6.63 & 5.60 & 6.82 & 5.13 & 4.93 & 4.26 & 3.28 & 2.78 \\
Kurtosis & 3.78 & 3.62 & 1.89 & 1.87 & 1.62 & 1.47 & 3.41 & 3.37 \\
\hline
\end{tabular}




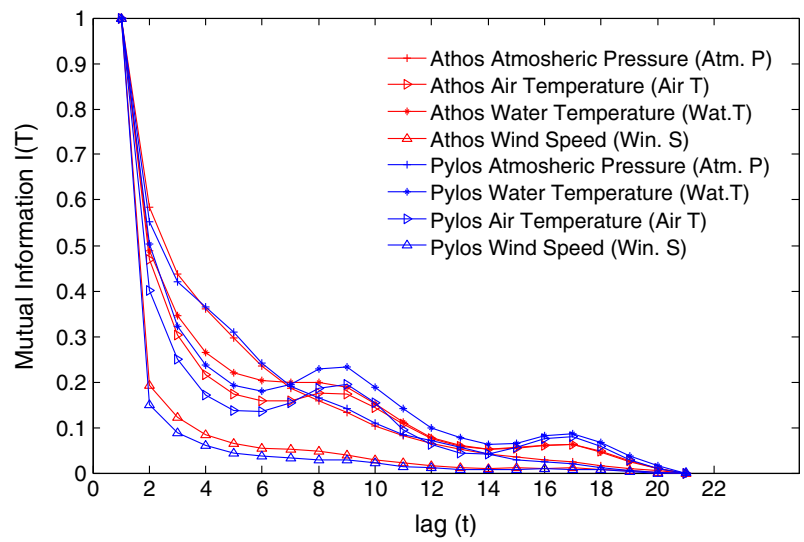

Fig. 3 Normalized mutual information of the time series from Athos station (red lines) and Pylos station (blue lines) (time lag is given in units $\Delta \mathrm{t}=3 \mathrm{~h}$ )

attain almost the same values and are sufficiently greater than 0.5 . These values indicate persistent behaviour and a long memory in the time series. We can see that Air and water temperature present the highest persistence while Atmospheric Pressure presents lower persistence followed by wind speed. These results are in qualitative agreement with the results of mutual information.

The results of Hjorth parameters mobility and complexity of the time series are also presented in Table 3. We can also see that for Win. S. time series Hjoth mobility and complexity present different behavior than the rest time series. Specifically, for Win. S time series, the Hjorth mobility is lower at Pylos than at Athos station and consequently the Hjorth complexity is higher at Pylos than at Athos stations.

\subsection{Spatiotemporal Dependencies}

In order to examine whether there exist correlations between observations, we employed the cross correlation function (ccf). Since there are many significant correlations at different time lags, we choose as the value of correlation, the first highest significant value either at positive or negative time lags. For instance, in Fig. 4a we present the result of the cross correlation function between Air Temperature and Water Temperature from Athos station. The blue lines indicate the confidence intervals $( \pm 2 / \sqrt{N}=0.0268)$. In the upper row, we present the time series and in the bottom the results of the cross correlation function. We can clearly see, that the first higher correlation is positive with a value $\operatorname{ccf}=0.92$ at a positive time lag of 23 . This means that the Air T time series leads Wat. T time series by almost 3 days (since it presents a

Table 3 Hurst exponent with R/S method and Hjorth mobility and complexity

\begin{tabular}{lllllllll}
\hline & $\begin{array}{l}\text { Athos } \\
\text { Win. S }\end{array}$ & Pylos & $\begin{array}{l}\text { Athos } \\
\text { Atm. P }\end{array}$ & Pylos & $\begin{array}{l}\text { Athos } \\
\text { Air. T }\end{array}$ & Pylos & $\begin{array}{l}\text { Athos } \\
\text { Wat. T }\end{array}$ & Pylos \\
\hline Hurst Exponent & 0.84 & 0.82 & 0.87 & 0.88 & 0.98 & 0.99 & 0.99 & 0.99 \\
Hjorth Mobility & 0.07 & 0.05 & 0.15 & 0.16 & 0.09 & 0.13 & 0.55 & 0.65 \\
Hjorth Complexity & 19.51 & 25.72 & 7.23 & 7.17 & 14.46 & 10.86 & 2.78 & 2.37 \\
\hline
\end{tabular}



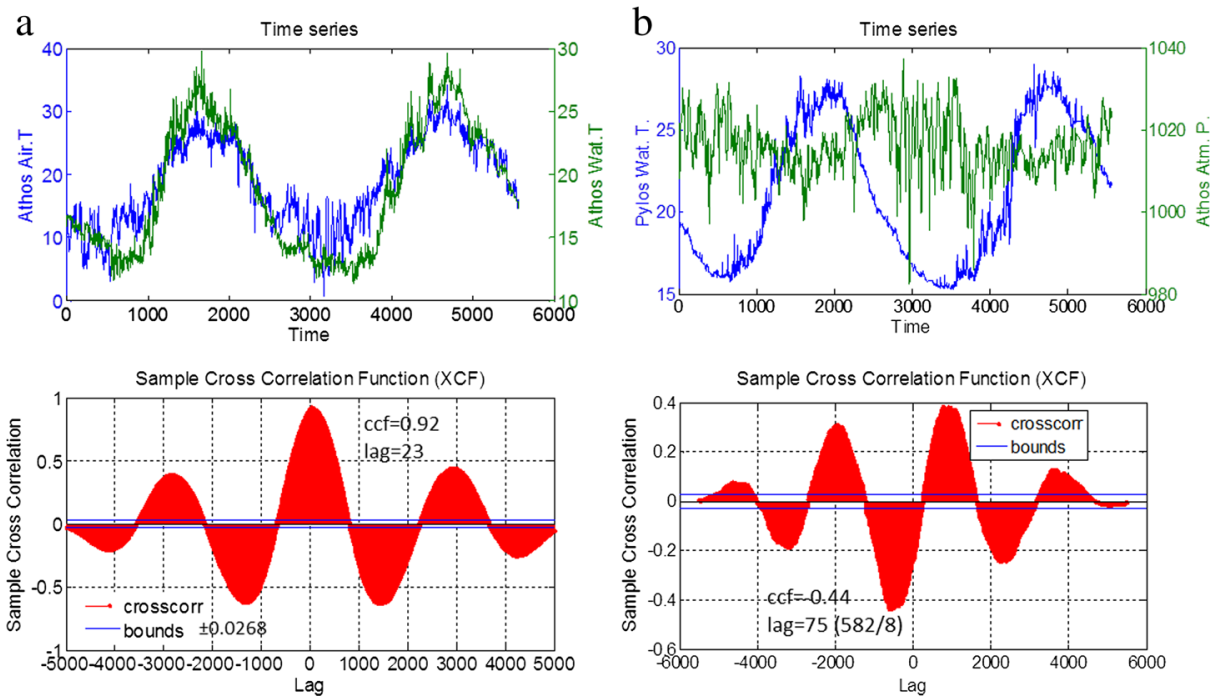

Fig. 4 The upper row shows the time series and the lower row shows the cross correlation as a function of time delay for a: a positive relationship and $\mathbf{b}$ for a negative relationship (time is in units of $\Delta \mathrm{t}=3 \mathrm{~h}$ ). The blue lines denote expected $95 \%$ confidence intervals $( \pm 0.0268)$

time lag 23 in units of $\Delta t=3 \mathrm{~h}$ ) and has a positive influence on Wat. T variable. Also, the result of the performance of the ccf function among Atmospheric Pressure from Athos station and Water Temperature from Pylos station are shown in Fig. 4b. In this case, we chose the first higher negative correlation with a value $\mathrm{ccf}=-0.44$ at a time lag $=75$. This means that the two variables present a negative correlation with positive time lag and Wat. T time series at Pylos station and leads Atm. P data at Athos station by almost 75 days (582/8), since the sampling interval was $3 \mathrm{~h}$ with an average over 10 min resulting in 8 values per day and both time series are negatively correlated.

Consequently, in Fig. 5a we present the results of cross correlation of the time series at Athos station and Pylos station at micro-scale level and at macro-scale level in order to
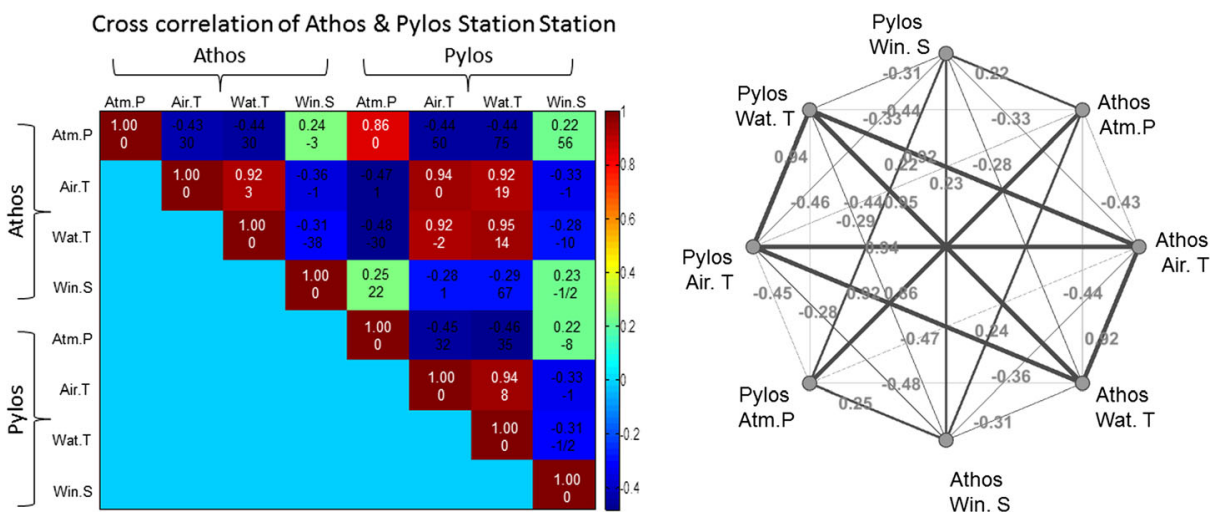

Fig. 5 Cross correlation of measurement variables both at local scale and at global scale among variables from Athos and Pylos station (time is in units of $\Delta \mathrm{t}=3 \mathrm{~h}$ ) 
examine whether Athos variables have any effect on the observed spatiotemporal dependencies on the variables from Pylos station and vice - versa.

In each cell, the upper value refers to the cross correlation value which if it is positive indicates a positive correlation between the variables, while if it is negative indicates a negative correlation. The lower value in the cell represents the correlation time (time lag) where the highest value of cross correlation occurs; if it is positive, indicates that the first variable (the variable that is on the row) leads the second variable (on the column). Figure 5b illustrates the representative constructed graph where the values of cross correlation are also indicated.

In general, from Fig. 5a it can be seen that both Athos and Pylos stations demonstrate almost the same behavior with respect to the values of cross correlation. We observe a negative correlation between Atmospheric Pressure and Air Temperature (ccf $=-0.43)$, Atmospheric Pressure and Water Temperature ( $c \mathrm{cf}=-0.44$ Athos $/ \mathrm{ccf}=-0.46$ Pylos), Air Temperature and Wind Speed ( $\mathrm{ccf}=-0.36$ Athos $/ \mathrm{ccf}=-0.33$ Pylos), and Water Temperature with Wind Speed $(\mathrm{ccf}=-0.31)$. At a micro-level, both at Athos and Pylos stations, there exist positive correlations with the strongest correlation structures between Air temperature and Water Temperature ( $\mathrm{ccf}=0.92$ Athos $/ \mathrm{ccf}=0.94$ Pylos). The lowest positive cross correlation occurs between Atmospheric Pressure and Wind Speed around the value of 0.22 to 0.24 time series both at local level (Athos or Pylos station).

Moreover, at a global scale we can observe that there are strong positive correlations among variables from Athos and Pylos station. Specifically, larger positive values of cross correlation can be observed between Atmospheric Pressure of Athos and Atmospheric Pressure of Pylos station (ccf=0.86), Air Temperature of Athos and Atmospheric Pressure of Pylos ( $\mathrm{ccf}=0.94)$, Air Temperature of Athos and Water Temperature of Pylos $(\mathrm{ccf}=0.92)$, Water Temperature of Athos and Air Temperature of Pylos (ccf=0.92), and finally, Water Temperature of Athos and Water Temperature of Pylos ( $c \mathrm{cf}=0.95)$. It is noted that also at global level the Air and Water Temperature are negatively correlated with Atmospheric Pressure and Wind Speed.

While the cross correlation values exhibit similar behavior, we do not observe the same behavior for the correlation time lags. In fact, we observe difference in correlation time lags, at the micro-scale (locally at each station) between Air T and Wat. T, where the time lag at Pylos station is three times the time lag of Athos. Another difference occurs between Wat. T and Win. $\mathrm{S}$ where at Athos station the Win. S leads Wat. T time series with a time lag of 38 days while at Pylos station the time lag is a half day.

Once the cross correlation among variables has been discussed, we can then proceed with the performance causality measures between the variable for identifying causal connectivity among them. Knowledge about causalities would be of great importance, since it provides information on the spatiotemporal dependencies. First, at a micro-scale level, the normalized results of the performance of Granger Causality measure are summarized in Fig. 6a-b for Athos and in Fig. 6c-d for Pylos station.

The direction of causalities is from columns to rows. We observe first at local scale that the Granger causality values are different at Athos and Pylos station. The analysis denotes that Wind Speed of Athos causes Air Temperature at Athos $(\mathrm{GC}=0.57)$, while there is no cause effect of Wind Speed of Pylos to Air Temperature of Pylos $(\mathrm{GC}=0.00)$. Moreover, we observe that there is a weak causality between Wind Speed of Pylos to Water Temperature at Pylos $(\mathrm{GC}=0.29)$, which does not occur at Athos station. Additionally, there is a cause from Water Temperature of Athos to Atmospheric Pressure of Athos $(\mathrm{GC}=0.16)$ which is not observed in Pylos $(\mathrm{GC}=0.00)$. However, the other values of causality among variables, are very similar. From Fig. 6e to d, where the Granger causalities are presented, it is of great interest to observe 

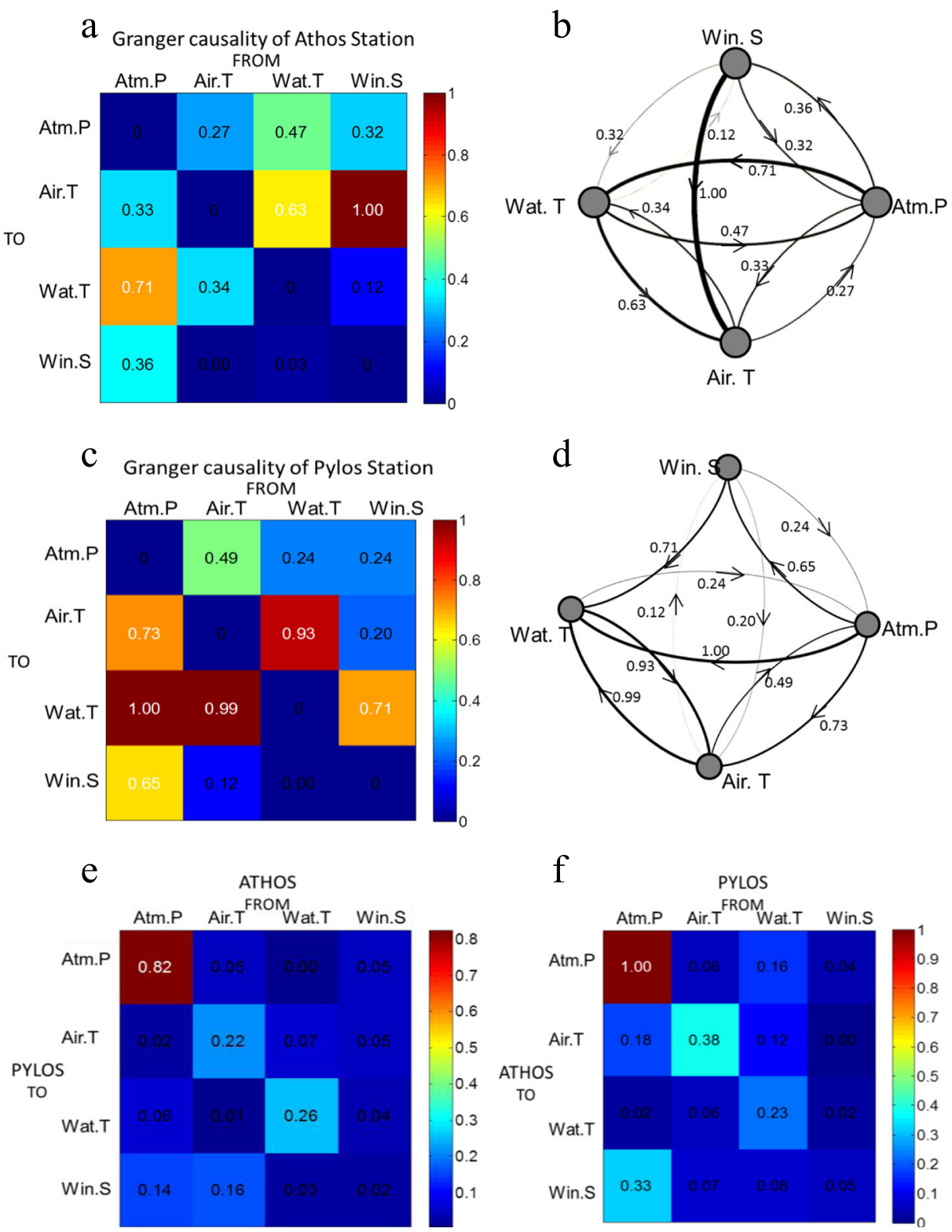

Fig. 6 Granger causality among variables from Athos and Pylos station

that the Granger causality value (GC) from Atmospheric Pressure of Pylos seems to affect the Atmospheric Pressure of Athos $(\mathrm{GC}=1.00)$ more than the Atmospheric Pressure of Athos the Atmospheric Pressure of Pylos $(\mathrm{GG}=0.82)$.

According to the theory of hypothesis testing to determine whether the result is statistically significant, significance values are calculated ( $\mathrm{p}$-values), which define the probability of impact since the null hypothesis is true. The null hypothesis is rejected if the p-value is less than the specified level of significance $(a=0.05)$. A statistically significant result is that in 
a

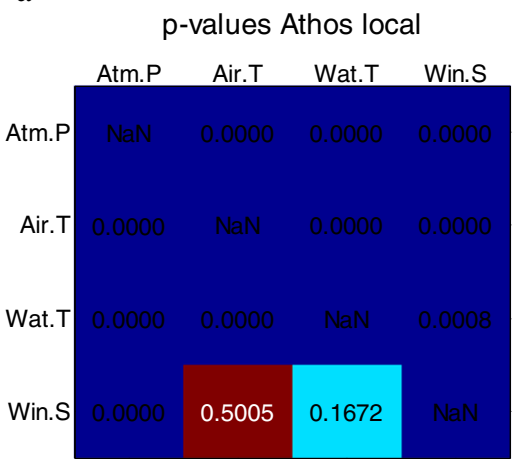

C

p-values Pylos local

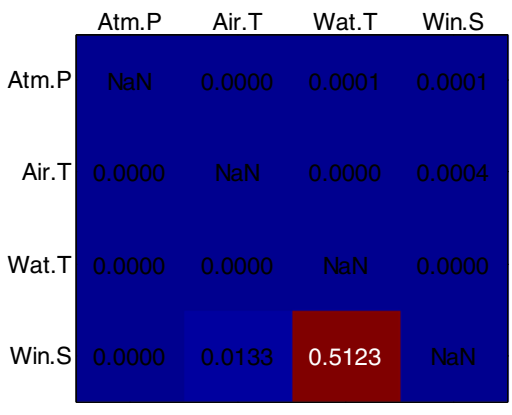

e p-values from Athos to Pylos

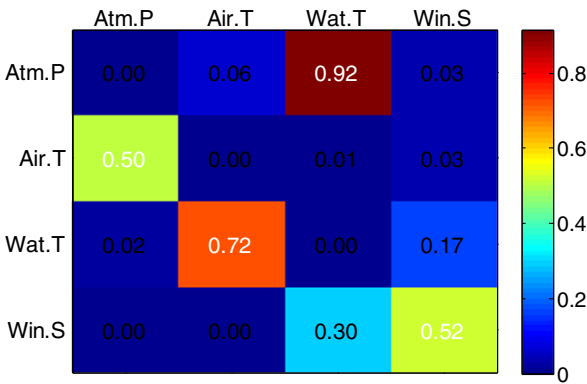

$\mathrm{g}$ significance level of $\mathrm{p}$-values 0.05 from Athos to Pylos
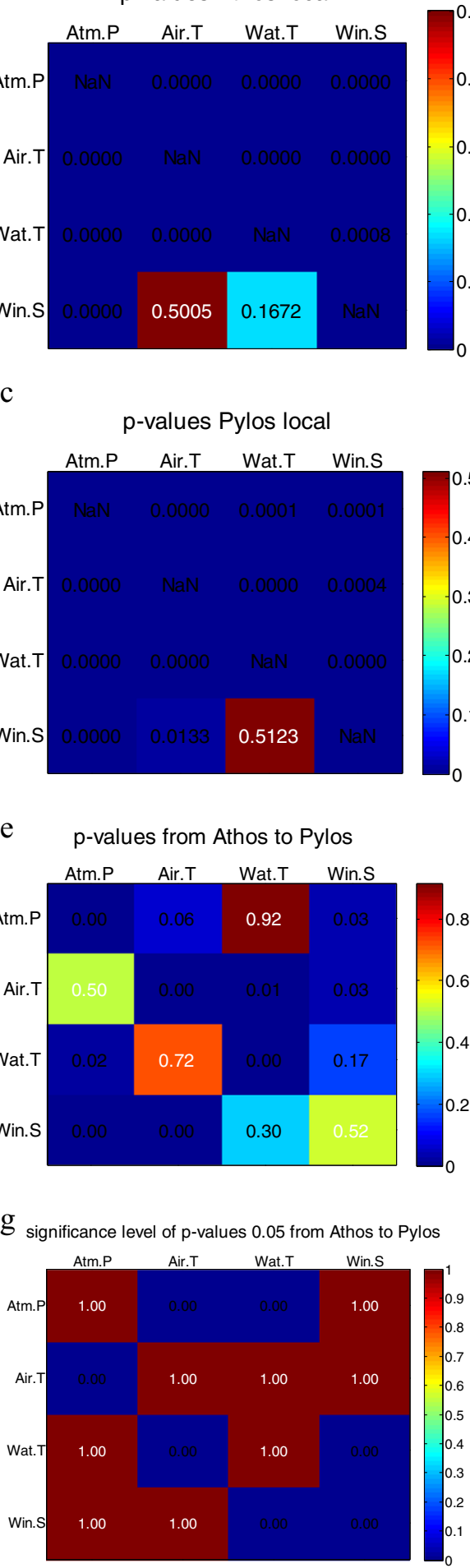

b

singificance level of $p$-values 0.05 Athos local

d singificance level of p-values 0.05 Pylos local

$\mathrm{f}$

f $p$-values from Pylos to Athos

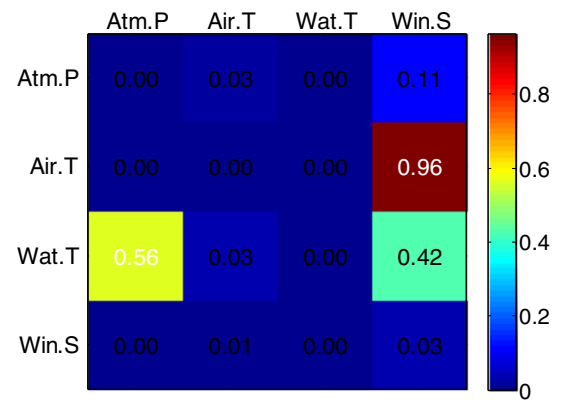

$\mathrm{h}$ significance level of $\mathrm{p}$-values 0.05 from Pylos to Athos

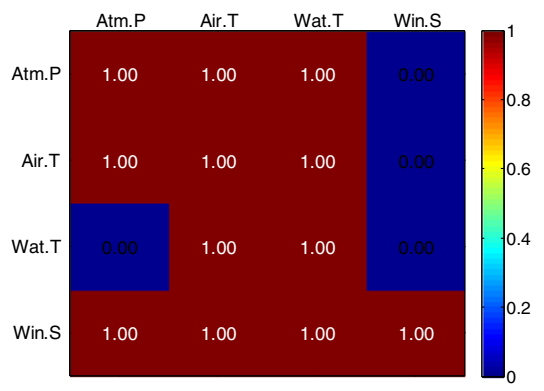


Fig. $7 \mathrm{P}$ values result, significance level of p-values (at $95 \%$ ) of Granger causality among variables, $\mathbf{a}-\mathbf{b}$ from Athos station, $\mathbf{b}-\mathbf{c}$ from Pylos station, e and $\mathbf{g}$ from Athos to Pylos station, and $\mathbf{f}$ and $\mathbf{g}$ from Pylos to Athos station

which the observed p-value is less than $5 \%$. We assume that there is no causality between the two time series (the null hypothesis Ho) by using the statistical test of Fisher distribution or as known the F-test on the alternative H1 (there is causality).

In the case of calculation of causality between the variables, the p-values by applying the criterion F-statistics (Barnett and Seth 2014) and the price level of significance using the criterion False Discovery Rate (FDR) (Benjamini and Hochberg 1995) shown in Fig. 7a-b for Athos station, Fig. 7c-d for Pylos station and Fig. 7e-h between observation from Athos to Pylos and vice-versa. The value 1 in the matrix of the Statistical significance level means that we can reject the null hypothesis (there is no causality among variables).

At Athos station, we can observe (Fig. 7a-b) that all the causalities between the variables are significant at a confidence level greater than $95 \%$, except the causality between Air T. and Win. S. and the causality between Wat. T. and Win. S. Moreover, in all the other cases at Pylos station we can reject the null hypothesis (no causality) apart from the causality between Wat. T. and Win. S. At a global scale (among variable from different stations) although the value of the causality is not high (Fig. 6e and f), summarizing the results of Fig. $7 \mathrm{~g}-\mathrm{h}$ we can generally observe that the variables from the area of Pylos influence the variables of Athos region more than they are influenced.

\section{Conclusions}

In this work data from Seawatch buoys at Aegean and Ionian Sea provided by Poseidon monitoring buoy for the period from January 2011 to November 2012 are analyzed with the purpose of understanding the underlying spatiotemporal characteristics of meteorological variables. As the understanding of the mechanisms could help improve the prediction of these systems, we focused on examining relationships and patterns at the micro-scale level (locally at each station) as well as at macro-scale level (globally between the stations). Apart from the performance of simple statistical measures and some linear and nonlinear indicators, we applied the cross correlation method in order to discover if changes at Athos station may outcome changes at Pylos station or vice versa. In general, the results show significant dependencies among variables indicating that the cross correlation analysis can be a powerful method, as there were significant positive and negative correlations between the data. Since the existence of correlation itself does not necessarily demonstrate the cause - effect relationship, we estimated the directionality of interactions among meteorological variables through the analysis of Granger causality based on prediction theory. We have shown that there is evidence of causal link among variables. Additionally, the results of the causality analysis have indicated that at a local scale there are some significant differences.

The proposed analysis could be extended to other spatiotemporal time series and extract more complex spatiotemporal dependencies between other physical variables too. Also, an important extension of this study is to incorporate the methodology of Complex Network analysis to the field of climate science (Ebbert-Uphoff 2012; Siggiridou et al. 2014; Hlinka et al. 2013). In the climate context, Complex Networks, constitute a powerful tool in order to identify hidden patterns of weather relationships and to analyze climate dynamics, through the analysis of networks topological measures, i.e., degree, clustering coefficient, average path length, diameter and modularity (Donges et al. 2009; Costa et al. 2011). 
Acknowledgments An initial version of the paper has been presented in the 12th International Conference on Protection and Restoration of the Environment, Skiathos Island, Greece, June 29 to July 3, 2014.

\section{References}

Attanasio A, Maravalle M, Fioravanti G (2012) Examining Granger causality between atmospheric parameters and radon. Nat Hazards 62:723-731

Attanasio A, Pasini A, Triacca U (2013) Granger causality analyses for climatic attribution. Atmos Clim Sci 3:515-522

Bagiorgas H, Mihalakou G, Rehman S, Al-Hadhrami L (2012) Offshore wind speed and wind power characteristics for ten locations in Aegean and Ionian Seas. J Earth Syst Sci 121:975-987

Barnett L, Seth A (2014) The MVGC multivariate Granger causality toolbox: a new approach to Granger-causal inference. J Neurosci Methods 223:50-68

Benjamini Y, Hochberg Y (1995) Controlling the false discovery rate: a practical and powerful approach to multiple testing. J R Stat Soc Ser B Methodol 57:289-300

Bilancia M, Vitale D (2012) Anthropogenic CO2 emissions and global warming: evidence from granger causality analysis. In: Ciaccio A, Coli M, Ibanez J (eds) Advanced statistical methods for the analysis of large datasets. Springer, New York, pp 229-240

Costa LF, Oliveira ON, Travieso G, Rodrigues FA, Villas Boas PR, Antiqueira L, Viana MP, Correa Rocha LEC (2011) Analyzing and modeling real-world phenomena with complex networks: a survey of applications. Adv Phys 60:329-412

Craigmile P, Guttorp P (2011) Space-time modelling of trends in temperature series. J Time Ser Anal 32:378-395

Darand M, Mansouri Daneshvar MR (2014) Regionalization of precipitation regimes in iran using principal component analysis and hierarchical clustering analysis. Environ Process 1:514-532

Donges J, Zou Y, Marwan N, Kurths J (2009) Complex networks in climate dynamics. Eur Phys J Spec Top 174: $157-179$

Drakopoulos P, Nittis K, Petihakis G, Kassis D, Pagonis P, Ballas D, Ntoumas M (2011) Monitoring chlorophyll concentrations with POSEIDON system's optical instruments. Proceedings of the Sixth International Conference on EuroGOOS, Poland

Ebbert-Uphoff I (2012) Causal discovery for climate research using graphical models. J Clim 25:5648-5665

Fraser A, Swinney H (1986) Independent coordinates for strange attractors from mutual information. Phys Rev A 33:1134-1140

Fritsch F, Carlson R (1980) Monotone piecewise cubic interpolation. SIAM J Numer Anal 17:238-246

Gaur A, Simonovic S (2015) Towards reducing climate change impact assessment process uncertainty. Environ Process 2:275-290

Granger C (1969) Investigating causal relations by econometric models and cross-spectral methods. Econometrica 37:424-438

Hjorth B (1970) EEG analysis based on time domain properties. Electroencephalogr Clin Neurophysiol 29:306-310

Hlinka J, Hartman D, Vejmelka M, Runge J, Marwan N, Kurths J, Paluš M (2013) Reliability of inference of directed climate networks using conditional mutual information. Entropy 15:2023-2045

Hurst H (1951) Long-term storage capacity of reservoirs. Trans Am Soc Civ Eng 116:770-808

Kassis D, Nittis K, Perivoliotis L (2013) Hydrodynamic variability based on the multi-parametric POSEIDON Pylos observatory of the south Ionian Sea. Ocean Sci Discuss 10:883-921

Kodra E, Chatterjee S, Ganguly A (2011) Exploring Granger causality between global average observed time series of carbon dioxide and temperature. Theor Appl Climatol 104:325-335

Kopasakis K, Georgoulas A, Angelidis P, Kotsovinos N (2012) Numerical modeling of the long-term transport, dispersion, and accumulation of Black Sea pollutants into the North Aegean coastal waters. Estuar Coasts 35:1530-1550

Korres G, Papadopoulos A, Katsafados P, Ballas D, Perivoliotis L, Nittis K (2011) A 2-year intercomparison of the WAM-Cycle4 and the WAVEWATCH-III wave models implemented within the Mediterranean Sea. Mediterranean Mar Sci 12:129-152

Kugiumtzis D (2013) Direct-coupling information measure from nonuniform embedding. Phys Rev E 87(062918):1-14

Mandelbrot B, Wallis J (1969) Robustness of the rescaled range R/S in the measurement of noncyclic long run statistical dependence. Water Resour Res 5:967-988

Meiling L, Xiangnan L, Aohui M, Ting L, Zhihong D (2014) Spatio-temporal stability and abnormality of chlorophyll-a in the Northern South China Sea during 2002-2012 from MODIS images using wavelet analysis. Cont Shelf Res 75:15-27

Nelson-Wong E, Howarth S, Winter D, Callaghan J (2009) Application of autocorrelation and cross correlation analyses in human movement and rehabilitation research. J Orthop Sports Phys Ther 39:287-295 
Nittis K, Zervakis V, Perivoliotis L, Papadopoulos A (2001a) Operational monitoring and forecasting in the Aegean Sea: system limitations and forecasting skill evaluation. Mar Pollut Bull 43:154-163

Nittis K, Zervakis V, Papageorgiou E, Perivoliotis L (2001b) Atmospheric and oceanic observations from the Poseidon buoy network: initial results. Glob Atmos Ocean Syst 8:87-99

Paluš M (2014) Cross-scale interactions and information transfer. Entropy 16:5263-5289

Papadopoulos A, Katsafados P (2009) Verification of operational weather forecasts from the POSEIDON system across the Eastern Mediterranean. Nat Hazards Earth Syst Sci 9:1299-1306

Papadopoulos A, Katsafados P, Kallos G, Nickovic S (2002) The weather forecasting system for POSEIDONan overview. Glob Atmos Ocean Syst 8:219-237

Papana A, Kyrtsou C, Kugiumtzis D, Diks C (2013) Simulation study of direct causality measures in multivariate time series. Entropy 15:2635-2661

Paruch AM, Mæhlum T, Robertson L (2014) Changes in microbial quality of irrigation water under different weather conditions in Southeast Norway. Environ Process 2:115-124

Pasini A, Triacca U, Attanasio A (2012) Evidence of recent causal decoupling between solar radiation and global temperature. Environ Res Lett 7:034020. doi:10.1088/1748-9326/7/3/034020

Probst W, Stelzenmüller V, Fock H (2012) Using cross correlations to assess the relationship between timelagged pressure and state indicators: an exemplary analysis of North Sea fish population indicators. ICES J Mar Sci 69:670-681

Seth A (2010) A MATLAB toolbox for Granger causal connectivity analysis. J Neurosci Methods 186:262-273

Siggiridou E, Kugiumtzis D, Kimiskidis V (2014) Correlation networks for identifying changes in brain connectivity during epileptiform discharges and transcranial magnetic stimulation. Sensors 14:12585-12597

Sigrist F, Künsch H, Stahel W (2012) A dynamic nonstationary spatio-temporal model for short term prediction of precipitation. Ann Appl Stat 6:1452-1477

Soukissian T, Chronis G, Nittis K (1999) POSEIDON: operational marine monitoring system for Greek seas. Sea Technol 40:31-37

Tastu J, Pinson P, Kotwa E, Madsen H, Nielsen A (2011) Spatio-temporal analysis and modeling of short-term wind power forecast errors. Wind Energy 14:43-60

Triacca U (2005) Is Granger causality analysis appropriate to investigate the relationship between atmospheric concentration of carbon dioxide and global surface air temperature? Theor Appl Climatol 81:133-135

Velaoras D, Lascaratos A (2010) North-Central Aegean Sea surface and intermediate water masses and their role in triggering the Eastern Mediterranean Transient. J Mar Syst 83:58-66

Velaoras D, Kassis D, Perivoliotis P, Pagonis P, Hondronasios A, Nittis (2013) Temperature and salinity variability in the Greek Seas based on POSEIDON stations time series: preliminary results. Mediterranean Mar Sci 14(3):5-18

Wiener N (1956) The theory of prediction. In: Beckenbach E (ed) Modern mathematics for engineers. McGrawHill, New York

Wilks S (1995) Statistical methods in the atmospheric sciences: an introduction. Academic, California 